\title{
Publisher Correction: Dive into the Universe
}

May Chiao

Correction to: Nature Astronomy https://doi.org/10.1038/s41550-020-01231-w, published online 24 September 2020.

The initially published version of this Books \& Arts article referred to Wolfgang Steffen as one of the pioneers of the type of 3D visualization outlined in the article, and suggested the technique was straightforward; neither points are accurate. The sentence "The general procedure, according to one of the pioneers, Wolfgang Steffen, is straightforward:" has therefore been amended to read "The general procedure, according to 3D astrophysical modelling expert Wolfgang Steffen, is straightforward (at least conceptually):". Furthermore, the cover image was mistakenly of the US edition instead of the UK edition; the correct image has been uploaded. All versions of the article have been corrected.

Published online: 13 October 2020

https://doi.org/10.1038/s41550-020-01251-6

๑) Springer Nature Limited 2020 